\title{
Solution processed reduced graphene oxide/metal oxide hybrid electron transport layers for highly efficient polymer solar cells
}

K. D. G. Imalka Jayawardena, Rhys Rhodes, Keyur Gandhi, M. R. Ranga Prabath, G. Dinesha M. R. Dabera, Michail J. Beliatis, Lynn Rozanski, S. J. Henley, S. Ravi P. Silva ${ }^{\text {a) }}$

Advanced Technology Institute, University of Surrey, Guildford, GU2 7XH, UK

${ }^{a}$ Electronic mail: s.silva@ surrey.ac.uk

\begin{abstract}
The solution to the all polymer solar cell that breaks the $10 \%$ efficiency barrier keeps getting closer, with ever more scrutiny on each component in the active device for better performance. Much effort has been expended on the hole transport layers and photo-active polymer blends in these solution deposited photovoltaic cells. In this study we examine the merits of incorporating solution deposited metal oxide and hybrid metal oxide/reduced graphene oxide (RGO) based electron transport layers (ETL) with a view to providing further improvements to the PV cell architecture. Low bandgap active layer blends of [3,4$b]$ thiophene/benzodithiophene (PTB7) and [6,6]-phenyl C70 butyric acid methyl fullerene $\left(\mathrm{PC}_{70} \mathrm{BM}\right)$ with efficiencies in excess of $7 \%$ are fabricated and the performance of four different ETL material systems based on $\mathrm{TiO}_{2}, \mathrm{ZnO}, \mathrm{TiO}_{2} / \mathrm{RGO}, \mathrm{ZnO} / \mathrm{RGO}$ are compared to thermally evaporated optimised reference bathocuproine (BCP) PV devices. Hybrid metal oxide/RGO ETL incorporated solution processed devices show an improved device performance compared to metal oxide only devices, with the performance comparable to the thermally evaporated $\mathrm{BCP}$ with fill factors of $68 \%$ and short circuit currents reaching 15 $\mathrm{mA} / \mathrm{cm}^{2}$. The enhanced performance of the RGO incorporated hybrid ETL points the way for novel transport layers for all solution processed devices.
\end{abstract}

\section{Body}

Growing concerns with regards to the diminishing supply of fossil fuels and the impact of such non-renewables on global warming has made solar energy generation an important area of research. Of the many types of solar-energy converting systems, organic photovoltaics have attracted significant interest due to their low cost, light weight and the printable nature on flexible substrates. ${ }^{1-2}$ Bulk heterojunction (BHJ) organic solar cells are one of the material systems extensively investigated for this purpose, where an electron donating conjugated polymer and an electron accepting small molecule fullerene are been blended to form the active material. ${ }^{1,3}$ Recent breakthroughs in the development of low bandgap polymers for harvesting of photons from the red $^{4}$ to the near infrared ${ }^{5}$.

The performance of BHJ organic solar cells is driven by the following key processes: (1) absorption of a significant fraction of photons from the solar spectrum, (2) generation of 
excitons and their dissociation at the donor (D) - acceptor (A) interface (giving rise to the internal quantum efficiency), (3) transport of the free charges towards contacts, and, (4) extraction of these charges at the contacts to generate useful power (the external quantum efficiency or IPCE). Processes (1)-(3) have been extensively investigated, with engineering of the interfacial charge transport layers receiving attention due to its importance in the light coupling $^{6}$ and charge extraction in these devices ${ }^{7}$.

Two primary techniques are at present used in the deposition of such charge extraction layers: thermal evapouration ${ }^{8}$ and solution processing ${ }^{9}$. Although the use of thermally evaporated calcium an electron transport layer ${ }^{4}$, bathocuproine (BCP), a small molecule, that can also be deposited through thermal evapouration has also been used as an ETL ${ }^{8,10}$. However, recent investigations have focused on solution processed metal oxide layers have emerged as an alternative to thermally deposited ETL ${ }^{11}$ Furthermore, such metal oxide based ETLs are known to act as an optical spacer under appropriate conditions, further enhancing the device performance ${ }^{4}$. This route has also offered enhanced device satiability due to the moisture and oxygen scavenging nature of the metal oxides $^{12}$.

In the research discussed here, the performance of polymer solar cells with a standard (or non-inverted architecture) based on the high performing $[3,4-b]$ thiophene/benzodithiophene (PTB7) low bandgap polymer utilizing thermally evaporated BCP or solution deposited metal oxides (namely, zinc oxide and titanium oxide) or metal oxide/RGO composites are examined. Metal oxides as the ETL ${ }^{6,11,13}$, especially for inverted OPVs with power conversion efficiencies exceeding $7 \%$ have been reported ${ }^{11}$. However to the best of our knowledge, the use of hybrid metal oxide/graphene composites as have not been reported by other groups and as such paves a new way for improving the performance of OPVs. Traditionally, the charge transfer between metal oxide nanostructures is governed by a hopping process which leads to an increase in the series resistance of the device upon its incorporation as an ETL, Although RGO is not expected to possess the same mean free path for charge transport as graphene due to defects in the structure, it is reasonable to expect that RGO is likely possess mean free paths sufficiently long enough for charge transfer between two nanocrystals. As a result the metal oxide/RGO nanohybrids warrents investigation as an electron transport layer,

The architecture used for the polymer solar cells in this work is highlighted in Figure 1(a) with the energy level alignment for the layers shown in Figure 1(b). While $\mathrm{ZnO}, \mathrm{TiO}_{2}$ and RGO are observed to have favourably inclined band alignments for electron extraction from the active layer, the lowest unoccupied molecular orbital (LUMO) of BCP is expected to inhibit electron extraction. Despite this unfavourable band alignment, BCP is known to be an efficient electron extraction layer, primarily due to intermediate states existing in the bandgap that favours selective electron extraction from the active layer. ${ }^{14}$ Furthermore, BCP is also known to act as an exciton blocking layer that prevents the quenching of excitons generated in the active layer as a result of diffusion towards the $\mathrm{Al}$ contact, a diffusion layer that acts to prevent contact between hot $\mathrm{Al}$ and $\mathrm{BCP}$ thereby leading to the formation of an Ohmic contact and an interface layer that prevents the unfavourable dipole formation between AL and fullereences that inhibits electron extraction. ${ }^{15}$ 
The surface morphologies of the different nanostructures/layers spin cast on the $\mathrm{PTB} 7 / \mathrm{PC}_{70} \mathrm{BM}$ active material is shown in Figures 1(c)-(f). Analysis of the surface roughness over a scan area of $3 \mu \mathrm{m} \times 3 \mu \mathrm{m}$ indicates values of $2.47 \mathrm{~nm}, 2.46 \mathrm{~nm}, 3.03 \mathrm{~nm}, 3.22 \mathrm{~nm}$ and $3.34 \mathrm{~nm}$ respectively for $\mathrm{BCP}$ ( $4 \mathrm{~nm}$ thick layer) $\mathrm{TiO}_{2}, \mathrm{ZnO} \mathrm{TiO}_{2} / \mathrm{RGO}$ and $\mathrm{ZnO} / \mathrm{RGO}$. This indicates with the exception of solution deposited $\mathrm{TiO}_{2}$, the deposition of oxides and metal oxide/RGO composites leads to a slight increase in the surface roughness in comparison to BCP. Although such an increase could be considered detrimental to device performance (eg. through the reduction of the fill factor due to increased leakage currents), the surface roughness noted is not significant enough to lead to a degradation of device characteristics.

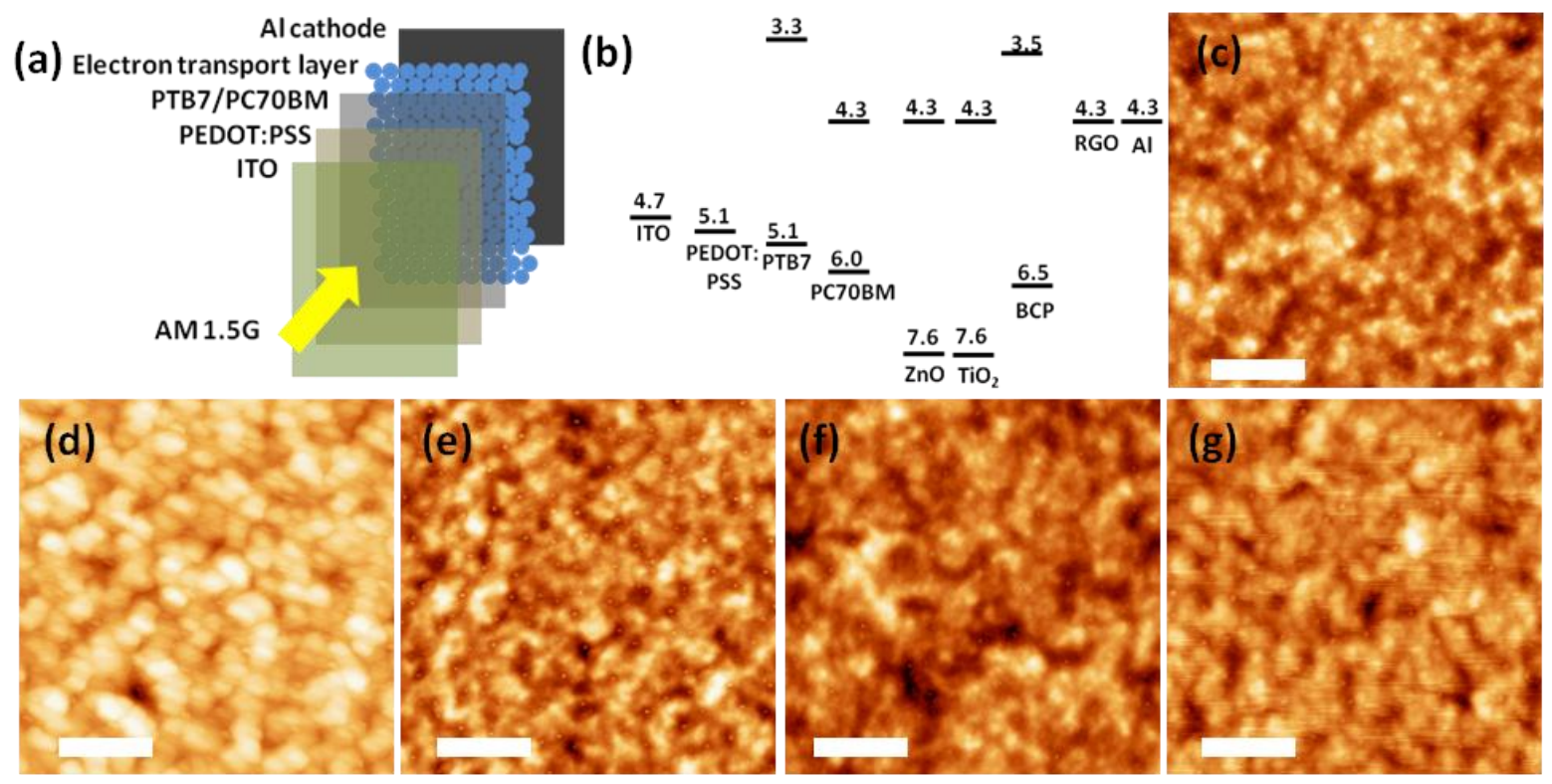

FIG. 1 (a) Schematic of the energy levels of ITO, PEDOT:PSS, PTB7, PC70BM, ZnO, $\mathrm{TiO}_{2}$, $\mathrm{RGO}, \mathrm{BCP}$ and $\mathrm{Al}$, (b) schematic of the device architecture employed, AFM microscans of (c) $4 \mathrm{~nm}$ BCP on PTB7:PC70BM, (d) ZnO on PTB7:PC70BM, (e) ZnO/RGO on PTB7:PC70BM, (f) $\mathrm{TiO}_{2}$ on PTB7:PC70BM and (g) $\mathrm{TiO}_{2} / \mathrm{RGO}$ on PTB7:PC70BM. The scale bars on figures (c)-(g) indicate $750 \mu \mathrm{m}$.

The performance of the PV devices (current density $(J)$-Voltage $(V)$ characteristics) incorporating the metal oxides, metal oxide/reduced graphene oxide and BCP under AM 1.5G $\left(100 \mathrm{mWcm}^{-2}\right)$ illumination are shown in Figure 2(a), with the device operation parameters given in Table 1 . The short circuit current density $\left(J_{s c}\right)$ obtained from the $J-V$ measurements are in agreement with those calculated using the IPCE spectra presented in figure 2(b). The performance of $\mathrm{PTB} 7 / \mathrm{PC}_{70} \mathrm{BM}$ bulk solar cells using solution processed $\mathrm{ZnO}, \mathrm{TiO}_{2}, \mathrm{ZnO} / \mathrm{RGO}, \mathrm{TiO}_{2} / \mathrm{RGO}$ as the ETL, are comparable to the performance obtained using thermally evaporated BCP. Further scrutiny of the device performance characteristics indicate that the utilisation of metal oxide/RGO nanohybrid layers is beneficial in terms of enhancing the power conversion efficiency in comparison to the metal oxide only devices. The enhanced performance is attributed to the reduced series resistance $\left(R_{s}\right)$ for the 
nanohybrid ETLs which leads to an increased charge extraction as observed through the imrpvement in the $\mathrm{J}_{\mathrm{sc}}$.

Unlike in the work reported by You et $\mathrm{al}^{11}$ (and also observed in our lab) for inverted device architecture, no UV irradiation of the metal oxide layers (especially $\mathrm{TiO}_{2}$ ) for efficient device performance was needed. Although the lack of a UV filter in the solar simulator used in this work could be ascribed as a possible reason, it is noted that the absorption due to the glass substrate and the presence of the photo-active polymer layers is likely to cut off a significant portion of the UV content. Therefore the observed high device performance close to record levels of $\sim 7.4-7.5 \%$ over relatively large areas $\left(\sim 30 \mathrm{~mm}^{2}\right)$ with off-the shelf solutions in the case of all the oxide based ETLs can be attributed to several different causes with the simplest being the doped nature of commercially available metal oxides. However, in the present case, it is more than likely that the deposition of hot $\mathrm{Al}$ species onto the metal oxides leads to the diffusion of the metal into the metal oxide resulting in a doped nanotrsucture which eliminates the need for any post UV treatment. As of present, further investigations are underway in order to obtain a better understanding of the actual physical process involved at the $\mathrm{Al} / \mathrm{ETL}$ interface.

Having understood that no post treatment is necessary to improve the charge extraction capability with the novel metal oxide and metal oxide/RGO nanohyrbid transport layers, we now proceed to obtain a fundamental understanding of these ETLs on the effect of charge extraction through Space Charge Limited Conduction (SCLC) analysis for electron only devices using he field independent Mott-Gurney equation ${ }^{16}$ :

$$
J=\frac{9}{8} \varepsilon_{r} \varepsilon_{0} \mu \frac{\left(V-V_{b i}\right)^{2}}{L^{3}}
$$

Where, $\varepsilon_{r}$ is the relative permittivity of the active layer which is taken to be $3.0, \varepsilon_{0}$ is the permittivity of free space, $V$ is the applied potential to the device, $V_{b i}$ is the built-in potential, $L$ is the device thickness (taken to be $100 \mathrm{~nm}$ ) and $\mu$ the charge mobility (in this case the electron mobility)of the device For calculation of $\mu$, the linear region of the $J \sim\left(V-V_{b i}\right)^{2}$ plots (Figure 2) was analysed under dark conditions. The built-in potential was estimated on the intersection point of the photo and dark characteristics of the electron only devices. ${ }^{17}$ The electron mobilities calculated based on the above analysis are also given in Table 1 . 

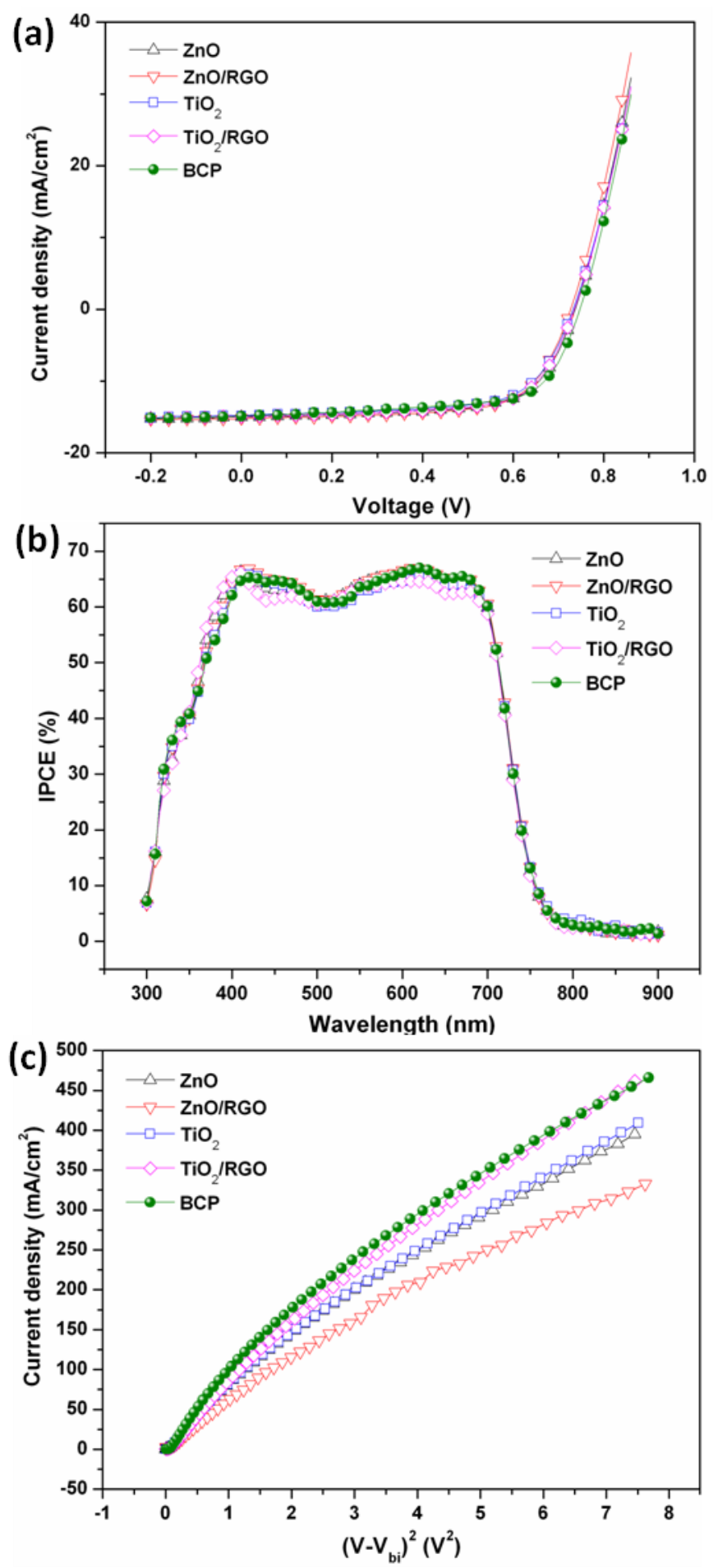

FIG. 2 (a) $J$ - $V$ characteristics of the photovoltaic devices fabricated using different ETLs, (b) the IPCE of the devices measured in (a) and (c) SCLC analysis for electron only devices incorporating $\mathrm{ZnO}, \mathrm{TiO}_{2}, \mathrm{ZnO} / \mathrm{RGO}, \mathrm{TiO}_{2} / \mathrm{RGO}$ and $\mathrm{BCP}$ as the ETL. 
Table 1: OPV performance parameters for different ETLs, with their the field independent electron mobility calculated using the Mott-Gurney equation.

\begin{tabular}{l|c|c|c|c|c|c}
\multicolumn{1}{c|}{$\mathrm{ETL}$} & $\mathrm{V}_{\mathrm{oc}}(\mathrm{V})$ & $\left.\mathrm{J}_{\mathrm{sc}}(\mathrm{mAcm})^{-2}\right)$ & $\begin{array}{c}\mathrm{FF} \\
(\%)\end{array}$ & $\eta$ & $\begin{array}{c}\mathrm{R}_{\mathrm{s}} \\
\left(\Omega / \mathrm{cm}^{2}\right)\end{array}$ & $\begin{array}{c}\mu \text { (field independent) } \\
\left(\mathrm{cm}^{2} \mathrm{~V}^{-1} \mathrm{~s}^{-1}\right)\end{array}$ \\
\hline $\mathrm{ZnO}$ & 0.74 & 14.90 & 66.9 & 7.39 & 5.24 & $5.3 * 10^{-4}$ \\
$\mathrm{ZnO} / \mathrm{RGO}$ & 0.72 & 15.19 & 68.6 & 7.50 & 4.92 & $3.7 * 10^{-4}$ \\
$\mathrm{TiO}_{2}$ & 0.74 & 14.84 & 65.7 & 7.22 & 5.47 & $4.9 * 10^{-4}$ \\
$\mathrm{TiO}_{2} / \mathrm{RGO}$ & 0.74 & 14.99 & 67.2 & 7.46 & 5.41 & $4.5 * 10^{-4}$ \\
$\mathrm{BCP}$ & 0.74 & 14.92 & 67.7 & 7.47 & 5.48 & $5.3 * 10^{-4}$
\end{tabular}

Electron mobility values calculated through the SCLC analysis for the electron only devices indicate values on the same order of magnitude similar to those reported on solution processed ETLs ${ }^{7}$ indicating that the transport layers used in this work (including the metal oxide/RGO nanohybrids) do not act in detrimental manner for charge extraction. Furthermore, it is noted here that the dark characteristics obtained for the electron only devices indicate an initial linear behaviour prior to the onset of SCLC indicating that an Ohmic onctant has been formed. However it is interesting to note that the RGO incorporated ETLs show a slightly lower mobility compared to metal oxide ETLs without RGO. As the $\mathrm{R}_{\mathrm{s}}$ for the ETLs is also observed to be reduced for the metal oxide/RGO nanohybrid ETL based devices, it is more than likely that the reduction of graphene oxide in the presence of the metal oxides has led to the doping of the oxide structures resulting in a low resistance ETL which enhances the photogenerated charge extraction. .

In conclusion, novel solution processable metal oxide/reduced graphene oxide nano-hybrid layers have been tested as electron transport layers for polymer solar cells. The results obtained indicates that the incorporation of graphene oxide to form reduced graphene oxides with the metal oxides leads to enhanced device performance over and above those observed for devices incorporating metal oxide (only) transport layers, indicating the beneficial nature of such complimentary systems for electron extraction. Furthermore, the performance of the devices incorporating these nano-hybrid layers is also observed to be comparable to the best performing devices utilizing thermally evaporated BCP as the ETL. Combined with the added advantage of silution processability, the high performances achieved with these nanohybrid layers indicate a promising route towards all solution processed polymer solar cells.

\section{Acknowledgements}

The authors would like to thank E.ON International Research Initiative and the Surrey Knowledge Transfer Account for funding the work described here. The authors are also grateful to EPSRC for the Postdoctoral Fellowships awarded (K. D. G. I. J. and M. J. B.), the University of Surrey for the Overseas Research Student Scholarship and the University Research Student Scholarships (ORS/URS) (G. D. M. R. D.) and the South East Physics Network for the Scholarship awarded (M. R. R. P.) 


\section{Experimental section}

Preparation of metal oxides and metal oxide/reduced graphene oxide composites: In this work, preparation of the $\mathrm{TiO}_{2}$ solutions were prepared using P25 samples from Degussa while $\mathrm{ZnO}$ nanocrystals were used as purchased from Fisher Scientific. In order to prepare the metal oxide/graphene composites, reduced graphene oxide was initially prepared using the method described by Hummers and Offeman. ${ }^{18}$ Preparation of the $\mathrm{TiO}_{2} / \mathrm{RGO}$ was carried out using P25 (Degussa) and the graphene oxide synthesized following the method described by Fan et al. ${ }^{19}$ while a similar procedure was followed using the commercially purchased $\mathrm{ZnO}$ for the preparation of $\mathrm{ZnO} / \mathrm{RGO}$ composites.

Device fabrication and characterization: Photovoltaic devices were fabricated on indium tin oxide (ITO) coated glass substrates (sheet resistance of $10 \Omega / \square$ ) precleaned using acetone and methanol followed by oxygen plasma ashing. PEDOT:PSS (Baytron PVP AI 4083) was then spin coated onto ITO and dried at $140^{\circ} \mathrm{C}$ for $15 \mathrm{~min}$ to form the hole transport layer. Subsequently, the blend of PTB7 (1-material)/ [6,6]-phenyl C70 butyric acid methyl fullerene (PC70BM, Solenne) $(25 \mathrm{mg} / \mathrm{ml}$ at $1: 1.5$ ratio $)$ in a mixed solvent system of chlorobenzene/1,8-diiodooctane (0.97:0.03) was spin coated on top of the hole transport layer to form an 80-100 $\mathrm{nm}$ thick active layer. This was followed by either spin coating of $\mathrm{ZnO}$, $\mathrm{TiO}_{2}, \mathrm{ZnO} / \mathrm{RGO}, \mathrm{TiO}_{2} / \mathrm{RGO}(\sim 30-50 \mathrm{~nm})$ or thermal evapouration of $\mathrm{BCP}(4 \mathrm{~nm})$ to form the ETL followed by the deposition of the Al cathode through thermal evapouration. The photovoltaic device performance of the above devices were characterized in air (without encapsulation) using a $300 \mathrm{~W}$ Xe Arc Lamp solar simulator (Abet Technologies) fitted with an Air Mass 1.5G Global (AM 1.5G) filter calibrated to an intensity if $100 \mathrm{~mW} / \mathrm{cm}^{2}$ and a Keithley 2425 sourcemeter. The IPCE measurements for the devices were carried out using a Bentham PVE300 system. For studies on the electron mobility based on space charge limited current (SCLC) analysis, electron only devices were fabricated by replacing the PEDOT:PSS layer in the architecture described above with a solution processed titanium suboxide layer $(\sim 5 \mathrm{~nm})$ deposited through spin coating of titanium isopropoxide precursor solution prepared using the technique described by Kim et al. ${ }^{6}$ and subsequent thermal treatment in air at $80^{\circ} \mathrm{C}$ for $10 \mathrm{~min}^{3}$. Except for the drying of PEDOT:PSS and the titanium suboxide layer, no other thermal treatment was carried following the deposition of the active layer is this was observed to lead to the degradation of the device performance.

\section{References}

1 Adikaari, A. A. D. T., Dissanayake, D. M. N. M. \& Silva, S. R. P. Organic-Inorganic Solar Cells: Recent Developments and Outlook. Ieee J Sel Top Quant 16, 1595-1606, doi:Doi 10.1109/Jstqe.2010.2040464 (2010).

2 Nismy, N. A., Jayawardena, K. D. G. I., Adikaari, A. A. D. T. \& Silva, S. R. P. Photoluminescence Quenching in Carbon Nanotube-Polymer/Fullerene Films: Carbon Nanotubes as Exciton Dissociation Centres in Organic Photovoltaics. Adv Mater 23, 3796-+, doi:DOI 10.1002/adma.201101549 (2011). 
3 Park, S. H. et al. Bulk heterojunction solar cells with internal quantum efficiency approaching 100\%. Nat Photonics 3, 297-U295, doi:Doi 10.1038/Nphoton.2009.69 (2009).

4 Liang, Y. Y. et al. For the Bright Future-Bulk Heterojunction Polymer Solar Cells with Power Conversion Efficiency of 7.4\%. Adv Mater 22, E135-+, doi:DOI 10.1002/adma.200903528 (2010).

5 Dou, L. T. et al. Tandem polymer solar cells featuring a spectrally matched lowbandgap polymer. Nat Photonics 6, 180-185, doi:Doi 10.1038/Nphoton.2011.356 (2012).

6 Kim, J. Y. et al. New architecture for high-efficiency polymer photovoltaic cells using solution-based titanium oxide as an optical spacer. Adv Mater 18, 572-+, doi:DOI 10.1002/adma.200501825 (2006).

7 He, Z. et al. Simultaneous Enhancement of Open-Circuit Voltage, Short-Circuit Current Density, and Fill Factor in Polymer Solar Cells. Adv Mater 23, 4636-4643, doi:10.1002/adma.201103006 (2011).

8 Nismy, N. A., Adikaari, A. A. D. T. \& Silva, S. R. P. Functionalized multiwall carbon nanotubes incorporated polymer/fullerene hybrid photovoltaics. Applied Physics Letters 97, doi:Artn 033105

Doi 10.1063/1.3464970 (2010).

9 Han, S. J., Adikaari, A. A. D. T., Jayawardena, K. D. G. I., Nismy, N. A. \& Silva, S. R. P. Control of nanocrystal surface defects for efficient charge extraction in polymerZnO photovoltaic systems. J Appl Phys 112, doi:Artn 066103

Doi 10.1063/1.4751432 (2012).

10 Vogel, M., Doka, S., Breyer, C., Lux-Steiner, M. C. \& Fostiropoulosa, K. On the function of a bathocuproine buffer layer in organic photovoltaic cells. Applied Physics Letters 89, doi:Artn 163501

Doi 10.1063/1.2362624 (2006).

11 You, J. et al. Metal Oxide Nanoparticles as an Electron-Transport Layer in HighPerformance and Stable Inverted Polymer Solar Cells. Adv Mater, n/a-n/a, doi:10.1002/adma.201201958 (2012).

12 Cho, S., Lee, K. \& Heeger, A. J. Extended Lifetime of Organic Field-Effect Transistors Encapsulated with Titanium Sub-Oxide as an 'Active' Passivation/Barrier Layer. Adv Mater 21, 1941-1944, doi:DOI 10.1002/adma.200803013 (2009).

13 Bai, S. et al. Inverted organic solar cells based on aqueous processed $\mathrm{ZnO}$ interlayers at low temperature. Applied Physics Letters 100, doi:Artn 203906

Doi 10.1063/1.4719201 (2012).

14 Jung, G. H., Hong, K., Dong, W. J., Kim, S. \& Lee, J. L. BCP/Ag/MoO3 Transparent Cathodes for Organic Photovoltaics. Adv Energy Mater 1, 1023-1028, doi:DOI 10.1002/aenm.201100411 (2011).

15 Gommans, H. et al. On the Role of Bathocuproine in Organic Photovoltaic Cells. Adv Funct Mater 18, 3686-3691, doi:DOI 10.1002/adfm.200800815 (2008).

16 Shrotriya, V., Yao, Y., Li, G. \& Yang, Y. Effect of self-organization in polymer/fullerene bulk heterojunctions on solar cell performance. Applied Physics Letters 89, doi:Artn 063505

Doi 10.1063/1.2335377 (2006).

17 Dissanayake, N. M. \& Zhong, Z. H. Unexpected Hole Transfer Leads to High Efficiency Single-Walled Carbon Nanotube Hybrid Photovoltaic. Nano Lett 11, 286290, doi:Doi 10.1021/N1103879b (2011).

18 Hummers, W. S. \& Offeman, R. E. Preparation of Graphitic Oxide. J Am Chem Soc 80, 1339-1339 (1958). 
19 Fan, W. Q., Lai, Q. H., Zhang, Q. H. \& Wang, Y. Nanocomposites of TiO2 and Reduced Graphene Oxide as Efficient Photocatalysts for Hydrogen Evolution. J Phys Chem C 115, 10694-10701, doi:Doi 10.1021/Jp2008804 (2011). 